\title{
Gastkommentar zum Beitrag „Frühgeburtenrate in 6 Perinatalzentren in Baden-Württemberg - Potential zur Reduktion der Frühgeborenenzahl“": Die schwierige Frage der Versorgungsqualität
}

\author{
Autor \\ Jens Maschmann \\ Institut \\ Medizinischer Vorstand, Universitätsklinikum Jena \\ Bibliografie \\ DOI https://doi.org/10.1055/a-0673-1826 \\ Z Geburtsh Neonatol 2018; 222: 210-211 \\ (c) Georg Thieme Verlag KG Stuttgart · New York \\ ISSN 0948-2393
}

Korrespondenzadresse

PD Dr. Jens Maschmann

Medizinischer Vorstand

Universitätsklinikum Jena

07740 Jena

Medizinischer.Vorstand@med.uni-jena.de
Die Autoren greifen mit ihrer Arbeit „Frühgeburtenrate in 6 Perinatalzentren in Baden-Württemberg - Potential zur Reduktion der Frühgeborenenzahl“ einen relevanten Aspekt der Perinatologie auf, da Morbidität und Mortalität invers mit dem Gestationsalter von Frühgeborenen korrelieren. Daher sind Strategien zu begrüßen und zu fördern, die zu einer Vermeidung (extremer) Frühgeburtlichkeit führen. Trotzdem wird es immer Konstellationen geben - sei es aus kindlicher oder mütterlicher Indikation - die es notwendig machen, eine Geburt zu forcieren. Die Autoren fordern als Schlussfolgerung ihrer Arbeit, dass die Frühgeborenenrate als Qualitätsindikator zur Beurteilung der Versorgungsqualität von Perinatalzentren mit aufgeführt werden sollte.

Allerdings wird dadurch die Beantwortung der schwierigen Frage nicht einfacher, wer denn eigentlich die Versorgungsqualität beurteilen soll und welche Schlussfolgerungen aus den Ergebnissen gezogen werden. Die Perinatal- und die Neonatalerhebung sind die beiden ältesten externen Qualitätssicherungsverfahren der Medizin in Deutschland. Mittlerweile wurden diese um eine öffentlich zugängliche Darstellung neonatologischer Ergebnisqualität (www. perinatalzentren.org) erweitert und stellen die bislang einzige verpflichtende öffentliche Darstellung medizinischer Ergebnisqualität in Deutschland dar.

Trotz Existenz der o.g. externen Qualitätssicherungsverfahren gelang es nicht, die Daten und Erfahrungen der vergangenen Jahrzehnte in Schlussfolgerungen oder gar ordnungsrechtliche Entscheidungen zur Verbesserung der Versorgungsqualität umzusetzen. Auch eine Anhebung der Mindestmengengrenze von 14 auf 30 sehr kleine Frühgeborene pro Jahr war mit den vorliegenden Daten nicht gerichtsfest zu untersetzen. Stattdessen wurde der 2005 gefasste Beschluss des Gemeinsamen Bundesausschusses (G-

1 Bericht zur Auswertung der Strukturabfrage 2017, IQTiG, https://perinatalzentren.org/strukturabfrage.php, 07.08.2018, 23:49h
BA) zu Strukturvorgaben an die peri- und neonatale Versorgung mit Einführung einer gestuften Versorgungsstruktur (Level 1, 2, perinatologischer Schwerpunkt und Geburtsklinik) zwischenzeitlich dermaßen verschärft, dass $91 \%$ aller Level 1 Perinatalzentren nach der jüngsten Erhebung des IQTiG 1 erklärt haben, die dort gestellten Anforderungen an die pflegerischen Anforderungen nicht erfüllen zu können. Gleichzeitig setzen die Kostenträger in den Budgetverhandlungen mit den Krankenhäusern das geltende Recht unerbittlich um, indem sie Einrichtungen, welche die Vorgaben aus dem G-BA-Beschluss nicht vollständig erfüllen, Budget abziehen. Auch die Meldungen von Einrichtungen gegenüber dem G-BA in 2017, bis Ende 2019 eine Übergangsfrist in Anspruch nehmen zu müssen, hat auf diese Budgetreduktion keinen Einfluss, obwohl Zielvereinbarungen auf Landesebene zur schrittweisen Zielerreichung abgeschlossen wurden. Konsequenterweise entwickelte sich bereits 2017 die absurde Situation, dass Zentren mit nachweislich hervorragender Ergebnisqualität Schwangere mit drohender Frühgeburt nicht aufgenommen haben ${ }^{2}$, um keine „Regelverletzung“ bei den G-BA-Vorgaben mit den o. g. Konsequenzen zu provozieren. Dies stellt aus meiner Sicht eine Perversion dar. In einem Bereich der Medizin, der wie kein anderer eine transparente und ausreichend risikoadjustierte Ergebnisqualität liefert, wird durch Strukturanforderungen eine potenzielle Verschlechterung der Versorgungssituation in Kauf genommen. Und dies obwohl das Thema Qualität schon seit der letzten Legislaturperiode politisch ganz groß geschrieben wird. Es ist ein mahnendes Beispiel für eine Entwicklung, in der gut gemeinte Vorgaben zur Qualitätsverbesserung kombiniert werden mit Vergütungsanreizen oder -kürzungen, die zu einer Verunsicherung und zu einem Dokumentationsaufwand führen, welche der Versorgungsqualität auf jeden Fall nicht förder-

2 Offener Brief des Universitätsklinikums Ulm vom 11.01.2018 zur Versorgungssituation in dessen Perinatalzentrum 2017 
lich sind. Einrichtungen werden weiterhin versuchen, sich regelund systemkonform zu verhalten, unabhängig davon, welche Anforderungen an Mindestmengen und Strukturanforderungen gestellt werden. Dass dabei Anreize gesetzt werden, medizinisch sinnvolles Vorgehen wie die Vermeidung von Frühgeburten zugunsten der Erreichung von Mindestmengen möglicherweise zu unterlaufen, sollte jedem normativ tätigen Akteur im Gesundheitswesen bewusst sein. Ein möglicher Ausweg könnte sein, konsequent die Mengen- und Strukturvorgaben auf ein Niveau zu bringen, bei dem es zu einer wirklichen Zentralisierung kommt, bei der dann auch inhaltlich sinnvolle Vorgehensweisen, wie die Verzögerung der Schwangerschaftsdauer bei Frühgeburtlichkeitsbestrebungen, nicht gleich zu einer Unterschreitung der Mindestmengen führt. Erforderlich dafür wäre allerdings ein politischer Durchsetzungswille, da es dann zu wirklich einschneidenden Veränderungen in der Versorgungslandschaft kommen würde. Diese hätten den Vorteil, dass sie für alle Akteure klar wären, währenddessen das bisherige Vorgehen zu eher erratischen Anpassungen führt.

Interessenkonflikt

Die Autoren geben an, dass kein Interessenkonflikt besteht. 